\section{Associação entre o estado nutricional antropométrico e a situação sócio-econômica de adolescentes em Niterói, Rio de Janeiro, Brasil}

\author{
Association between anthropometric nutritional \\ status and socioeconomic conditions among \\ adolescents in Niterói, Rio de Janeiro State, Brazil
}

\begin{abstract}
1 Escola Nacional de Ciências Estatísticas, Rio de Janeiro,

Brasil.

2 Instituto Nacional de

Câncer, Rio de Janeiro, Brasil.

${ }^{3}$ Escola Nacional de Saúde

Pública Sergio Arouca,

Fundação Oswaldo Cruz,

Rio de Janeiro, Brasil.

${ }_{4}$ Departamento de Nutrição

Social, Universidade Federal

Fluminense, Niterói, Brasil.
\end{abstract}

Correspondência

F. S. Gomes

Escola Nacional de Ciências

Estatísticas.

C. P. 7107, Rio de Janeiro, RJ 20230-972, Brasil.

fabiodasilvagomes@gmail.com

\section{Abstract}

This study focused on the relationship between nutritional and socioeconomic status among adolescents in Niterói, Rio de Janeiro State, Brazil. Data from 523 adolescents living in 1,734 households were collected from January to December 2003, from a total of 71,922 adolescents living in Niterói. Subjects were selected through a probabilistic household sample. The analyses included the estimation of confidence intervals for prevalence ratios and proportional distributions and independence tests between categories of nutritional status and per capita family income and number of residents per household. There was a significant positive association between underweight/thinness and number of residents in the same household (male: $p<$ 0.05 ; female: $p<0.001$ ). Number of residents in the same household was positively associated with prevalence of underweight/thinness in adolescents. Overweight/obesity was positively associated with per capita family income.

Nutritional Status; Nutrition Assessment; Nutrition Surveys; Socioeconomic Factors; Adolescent
Fabio da Silva Gomes 1,2

Luiz Antonio dos Anjos 3,4

Mauricio Teixeira Leite de Vasconcellos 1

\section{Introdução}

Uma revisão sistemática recente concluiu que a obesidade nos países em desenvolvimento não mais deveria ser considerada uma doença restrita somente a grupos de melhor situação sócio-econômica 1. De fato, associações inversas entre situação sócio-econômica e obesidade, entre mulheres, têm sido reportadas por diversos estudos em diferentes países 1,2,3,4,5.

Grupos populacionais de baixo status sócioeconômico têm sido mais atingidos pelo sobrepeso/obesidade no Brasil 6,7,8 e em outros países 7,8, com observação de associação inversa entre obesidade e estado sócio-econômico entre as mulheres 1. Dados recentes de adultos de Niterói confirmam tal associação: a prevalência de sobrepeso e obesidade foi maior nas mulheres de menor escolaridade e foi-se reduzindo com o aumento de escolaridade 9 . Entre os homens, por sua vez, foi observado um aumento na prevalência de sobrepeso/obesidade com o aumento da escolaridade 9 .

A obesidade na infância e adolescência também já é reconhecidamente um sério problema de saúde pública ${ }^{10}$. Investigações referentes a esse período do ciclo vital têm reportado altas prevalências de obesidade, e essas taxas têm-se apresentado crescentes 11 .

O sobrepeso e a obesidade têm sido reportados como as mais preocupantes inadequações nutricionais. No entanto, apesar dos focos e es- 
forços estarem voltados principalmente para a identificação e combate dessa condição e seus possíveis desfechos relacionados à saúde, as deficiências crônicas de energia e proteína, bem como carências de micronutrientes, apresentam-se como um agravo a ser cuidadosamente tratado.

Estudos realizados na cidade do Rio de Janeiro 12 e no Brasil 13 indicam queda da desnutrição entre os adolescentes e um preocupante aumento na prevalência de sobrepeso/obesidade entre esse grupo, nas últimas décadas 14 . No entanto, a relação do estado nutricional de adolescentes com suas condições sócio-econômicas tem sido pouco explorada, principalmente em países em desenvolvimento. Assim, o presente trabalho foi desenvolvido com o objetivo de investigar a relação entre o estado nutricional e a situação sócioeconômica familiar numa amostra probabilística de adolescentes residentes em Niterói.

\section{Métodos}

Os dados utilizados neste artigo foram coletados entre os meses de janeiro e dezembro de 2003, por meio da Pesquisa de Nutrição, Atividade Física e Saúde (PNAFS), a primeira, de base populacional, realizada com o objetivo de conhecer o estado nutricional e as condições de saúde de adolescentes e adultos residentes no Município de Niterói.

A população adolescente foi caracterizada segundo a faixa etária compreendida entre os 10 anos de idade completos e os 20 anos incompletos 15. Os dados explorados neste artigo incluem: medidas antropométricas (massa corporal e estatura) e informações sócio-demográficas (renda familiar per capita e número de moradores do domicílio), além de informações obtidas no próprio domicílio.

A massa corporal foi medida somente uma vez (com precisão de $0,1 \mathrm{~kg}$ ). A estatura foi obtida seguindo a padronização de Lohman et al. 16, durante apnéia pós-expiração; essa aferição foi realizada duas vezes, sendo a sua média usada nas análises. Ambas as medidas foram então utilizadas para classificar o estado nutricional antropométrico dos adolescentes a partir do cálculo do índice de massa corporal (IMC) definido como a divisão da massa corporal $(\mathrm{kg})$ pelo quadrado da estatura $\left(\mathrm{m}^{2}\right)$. Os dados das adolescentes grávidas ou lactantes, dos adolescentes sem membros ou com membros engessados, ou que estivessem usando calçados no momento da medição, não foram computados para análise no presente estudo. As informações sócio-demográficas foram colhidas por meio de um questionário padronizado.
A pesquisa foi conduzida dentro dos padrões exigidos pela Declaração de Helsinki e foi aprovada pelo Comitê de Ética em Pesquisa da Escola Nacional de Saúde Pública Sergio Arouca, da Fundação Oswaldo Cruz.

Para garantir a representatividade da população de Niterói, desenhou-se uma amostra probabilística de domicílios, selecionada em dois estágios (setor censitário e domicílio), conforme descrito em Bossan et al. 9 .

O procedimento de expansão da amostra da PNAFS baseou-se no produto das probabilidades de inclusão na amostra em cada estágio, utilizando o estimador proposto por Haldane 17, adaptado para uso em pesquisas domiciliares 18 . Assim, o peso natural do desenho corresponde ao inverso do produto das probabilidades de inclusão em cada estágio. O plano amostral empregado resultou em uma amostra composta por 1.734 domicílios, nos quais 523 moradores adolescentes foram avaliados.

Para reduzir os vieses de seleção, comuns em pesquisas domiciliares, os pesos naturais do desenho da amostra foram calibrados para gerar estimativas coincidentes com totais populacionais conhecidos 19, totalizando 71.922 adolescentes representados pela amostra (masculino $=35.812$; feminino $=36.110$ ). A proporção de adolescentes em cada classe de estado nutricional foi estimada segundo os valores críticos de IMC para idade e sexo recomendados pela OMS 20.

Para avaliar se a distribuição proporcional de classes de estado nutricional dentre as classes de renda familiar per capita e dentre as faixas de número de moradores do domicílio diferem significativamente foi utilizado o teste de homogeneidade baseado na estatística de Wald.

Para testar a independência entre cada uma das classes de estado nutricional e os quintos de renda familiar per capita ou as faixas de número de moradores do domicílio foi utilizada a estatística $\chi^{2}$ de Pearson, com ajustes de Rao-Scott de primeira e segunda ordem 21 .

Adicionalmente, foram estimados intervalos de 95\% de confiança (IC95\%) de razões de prevalência de adolescentes pertencentes às distintas classes de estado nutricional, segundo todos os possíveis pares de quintos de renda familiar per capita.

Consideraram-se as informações estruturais do plano amostral com peso calibrado para todas as análises; os resíduos da calibração também foram incorporados na estimação da variância e estatísticas derivadas dessa.

As análises foram realizadas em linguagem e ambiente R versão 2.6.1 (The R Foundation for Statistical Computing, Viena, Áustria; http:// www.r-project.org) sendo adotado um nível de 
significância de $5 \%(\alpha=0,05)$ para todos os testes estatísticos.

\section{Resultados}

A obesidade entre adolescentes moradores de Niterói aparece em maiores proporções nos 1o e 2o quintos de renda familiar per capita, e as proporções de adolescentes com sobrepeso seguem um gradiente de aumento do 1o para o 5 o quinto. Analisando o sobrepeso/obesidade em conjunto, nota-se que as proporções se assemelham entre os três primeiros quintos (1o, 2o e 3o); a partir do 4 o quinto, há uma tendência de aumento. Por sua vez, o baixo peso/magreza aparece em maior proporção no 1o quinto de renda familiar per capita, seguido do 3ㅇ, 2o e 4o quintos, consecutivamente, e é extinto no 5o quinto (Figura 1).

Entre os adolescentes do sexo masculino, a contribuição da proporção de indivíduos obesos na distribuição dentre os quintos é maior no $2 \underline{\text { o }}$ quinto, seguida do 5o e 1o quintos consecutivamente; a proporção dos adolescentes desse domínio com sobrepeso no 5o quinto é duas vezes maior que a proporção encontrada nos 1ㅇ e 2으 quintos juntos $[28,38 /(8,18+4,88)=2,17]$. O sobrepeso/obesidade analisado conjuntamente ocorre em proporções semelhantes nos dois primeiros quintos de renda (1o e 2o ), cai à metade no 3o quinto, e em seguida volta a aumentar a partir do 4 o quinto, atingindo a maior proporção no $5 \underline{\text { o }}$ quinto. As proporções de adolescentes do sexo masculino com baixo peso/magreza distribuemse de forma semelhante à apresentada para o total, exceto pela proporção no terceiro quinto (Figura 2).

Já entre as adolescentes, a proporção de indivíduos com baixo peso/magreza não ocorre a

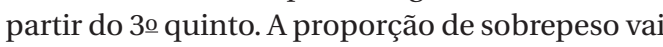
aumentando até o 4o quinto, decrescendo no último. As proporções de sobrepeso/obesidade em conjunto apresentam uma tendência de aumento do 1 o até o 4 o quinto de renda, no qual ocorre

Figura 1

Distribuição percentual da população de adolescentes moradores de Niterói, Rio de Janeiro, Brasil, segundo o estado nutricional classificado de acordo com a recomendação da Organização Mundial da Saúde (OMS) 20, por quintos de renda familiar per capita*.

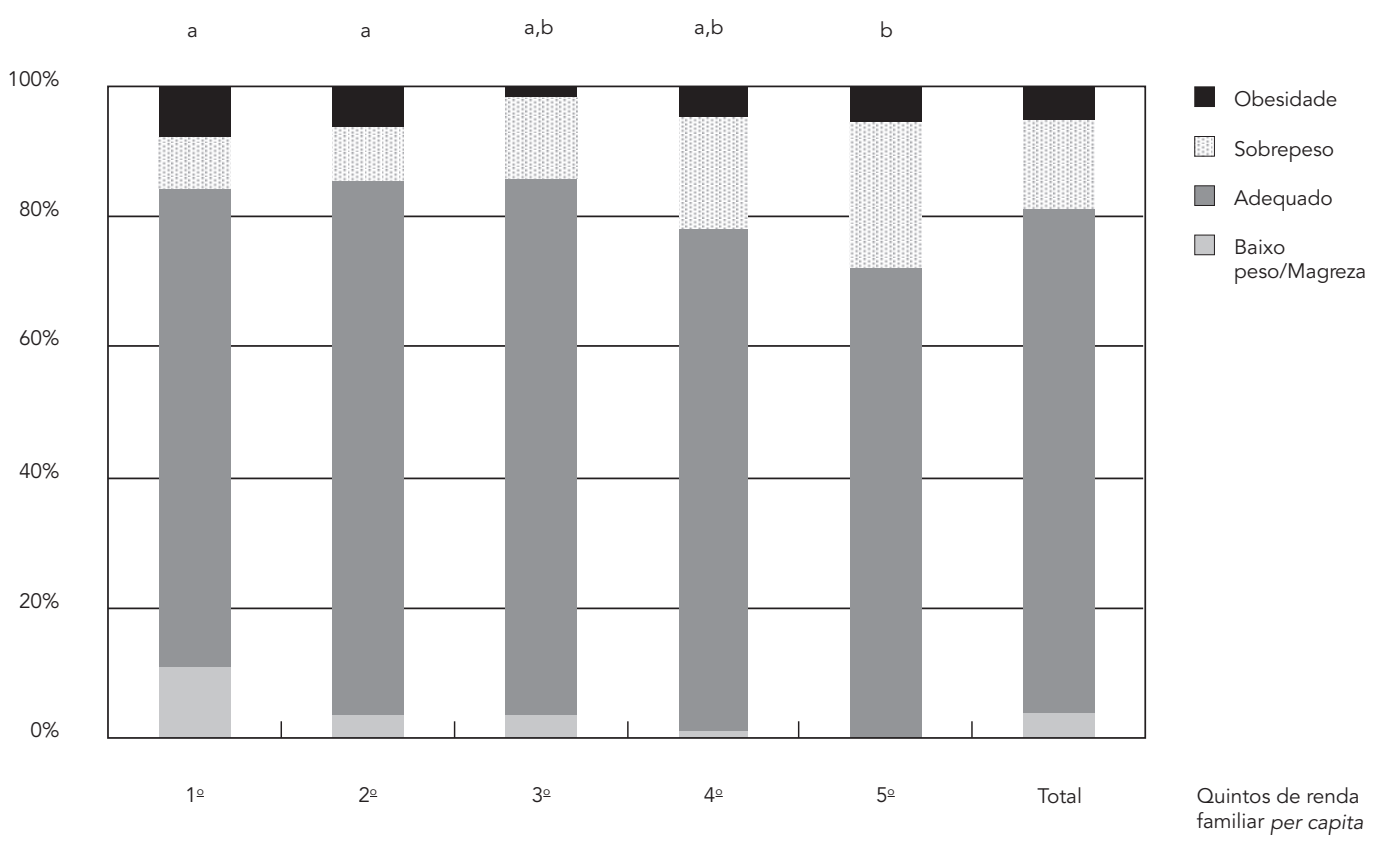

\footnotetext{
* Na comparação dois a dois entre os quintos de renda, a mesma letra na parte superior da barra indica que não há diferença significativa $(p>0,05)$ e letras distintas indicam diferenças significativas entre as distribuições proporcionais.
} 
Distribuição percentual da população de adolescentes do sexo masculino residentes em Niterói, Rio de Janeiro, Brasil, segundo o estado nutricional classificado de acordo com a recomendação da Organização Mundial da Saúde (OMS) 20, por quintos de renda familiar per capita *

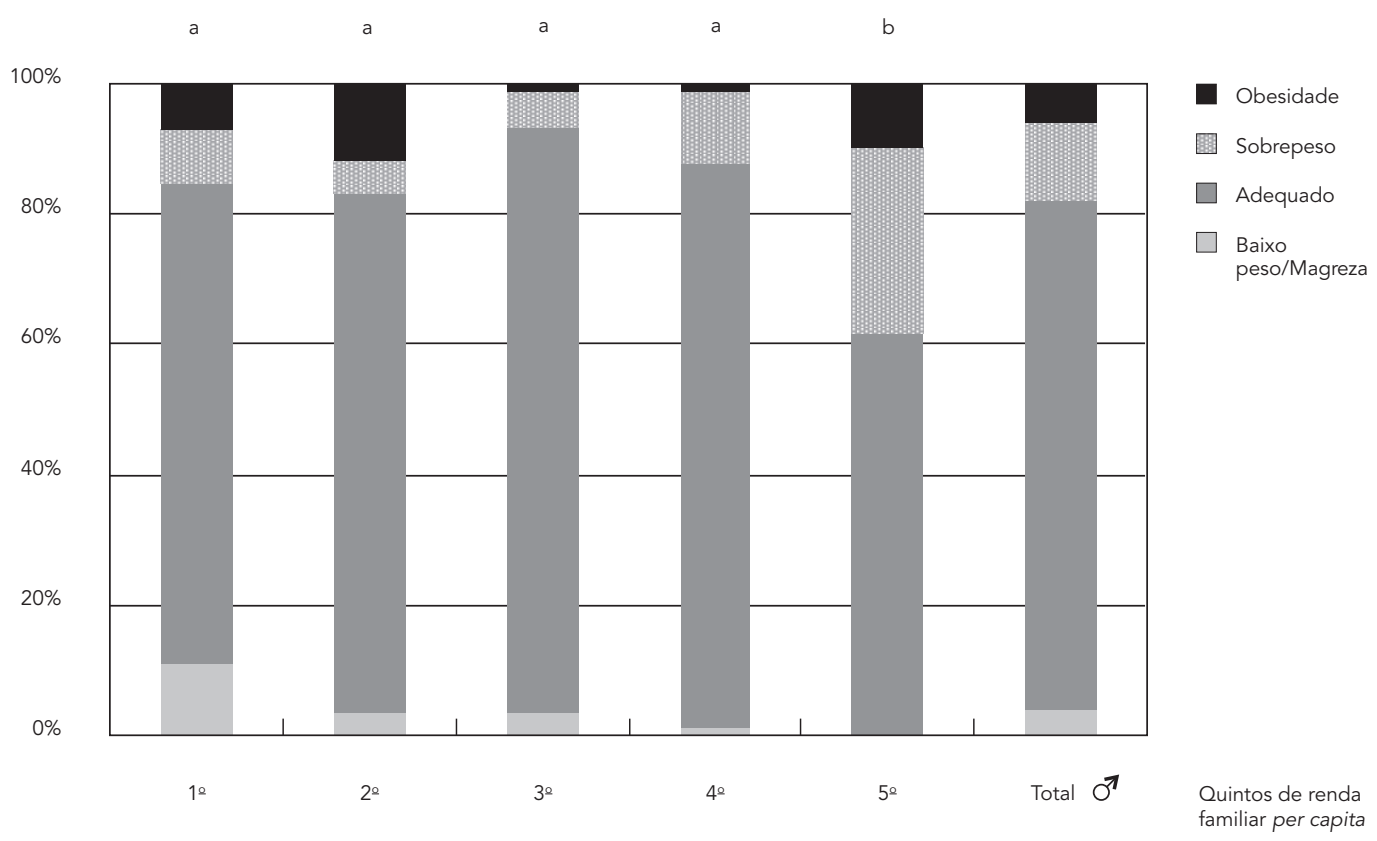

* Na comparação dois a dois entre os quintos de renda, a mesma letra na parte superior da barra indica que não há diferença significativa $(p>0,05)$ e letras distintas indicam diferenças significativas entre as distribuições proporcionais.

a maior proporção. Entretanto, a proporção no 5o quinto não acompanha essa tendência e se assemelha à do 1o quinto (Figura 3).

Ao testar formalmente a diferença entre as distribuições das proporções de classes do estado nutricional pelos quintos de renda, empregando a estatística de Wald para testar homogeneidade entre todos os possíveis pares de vetores, é possível identificar que, para ambos os sexos, a distribuição das classes de estado nutricional no 5 o quinto difere significativamente das distribuições no 1o e 2o quintos ( $\mathrm{p}<0,05$, para os dois pares de comparação), as quais não diferem significativamente das encontradas nos $3 \underline{\text { o }}(\mathrm{p}=0,09$ e p $=0,35$, em relação ao 1 o e 2 o quintos, respectivamente) e 4 o $(p=0,22$ e p $=0,37$, em relação ao 1o e 2o quintos, respectivamente) (Figura 1). Para o sexo masculino, o teste indicou que é significante a diferença entre o 5 o quinto e os demais $(\mathrm{p}<0,05)$ (Figura 2$)$. No caso das adolescentes, as distribuições de proporções dos quintos não diferiram significativamente $(\mathrm{p}$ >
0,05; para todos os possíveis pares de comparação) (Figura 3).

Testando a independência entre o estado nutricional e os quintos de renda familiar per capita, a estatística de Pearson, com correções de primeira e segunda ordem, revelou que há associação significativa entre esses fatores no grupo como um todo $(\mathrm{p}<0,05)$ e no sexo masculino $(\mathrm{p}<0,05)$, mas não entre as meninas $(\mathrm{p}=0,12)$. No entanto, avaliando separadamente cada uma das classes de estado nutricional, verificou-se que não há associação significativa entre cada uma das classes e a renda familiar per capita. Isso foi confirmado ao verificar que todos os IC95\% estimados para as razões de prevalência de cada uma das classes nutricionais entre os quintos continham o 1 (um) (Tabela 1).

Como uma proxy da situação sócio-econômica das famílias dos adolescentes, foi investigada a variável classe do número de moradores do domicílio. A Figura 4 revela que as prevalências por estado nutricional não diferem nas três pri- 
Distribuição percentual da população de adolescentes do sexo feminino residentes em Niterói, Rio de Janeiro, Brasil, segundo o estado nutricional classificado de acordo com a recomendação da Organização Mundial da Saúde (OMS) 20, por quintos de renda familiar per capita *.

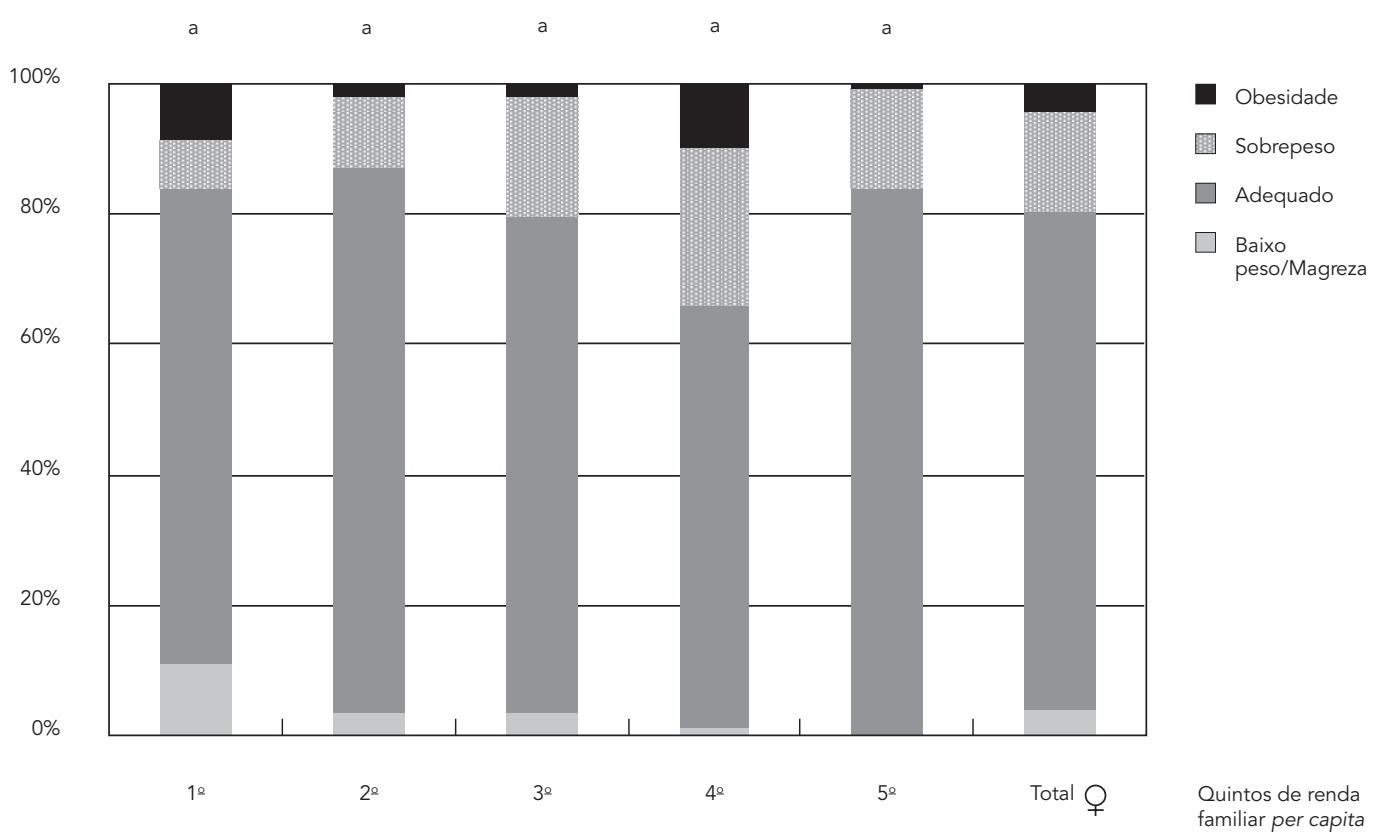

* Na comparação dois a dois entre os quintos de renda, a mesma letra na parte superior da barra indica que não há diferença significativa $(p>0,05)$ e letras distintas indicam diferenças significativas entre as distribuições proporcionais.

Tabela 1

Intervalos de 95\% de confiança das estimativas de razões de prevalência (IC95\% ${ }_{\mathrm{RP}}$ ) de adolescentes pertencentes às distintas classes de estado nutricional definidas pela Organização Mundial da Saúde (OMS) 20, segundo todos os possíveis pares de quintos de renda familiar per capita.

\begin{tabular}{|c|c|c|c|c|}
\hline \multirow{2}{*}{$\begin{array}{l}\text { Pares de quintos de renda } \\
\text { familiar per capita }\end{array}$} & \multicolumn{4}{|c|}{ Estado nutricional (IC95\% $\left.{ }_{\mathrm{RP}}\right)$} \\
\hline & Baixo peso & Eutrófico & Sobrepeso & Obesidade \\
\hline $1 \underline{0}: 2 \underline{o}$ & $-2,51 ; 8,97$ & 0,$74 ; 1,11$ & $-0,88 ; 2,87$ & $-1,27 ; 3,85$ \\
\hline 1ㅁ:3oㅡ & $-2,65 ; 8,95$ & 0,$76 ; 1,15$ & $-0,58 ; 1,97$ & $-13,33 ; 23,98$ \\
\hline $1 \underline{0}: 4 \underline{0}$ & $-13,37 ; 32,84$ & 0,$78 ; 1,17$ & $-0,39 ; 1,34$ & $-1,51 ; 4,78$ \\
\hline $1 \underline{0}: 5 \underline{0}$ & ne & 0,$84 ; 1,26$ & $-0,91 ; 1,65$ & $-1,34 ; 4,28$ \\
\hline 2ㅇ: $3 \underline{o}$ & $-3,41 ; 5,36$ & 0,$84 ; 1,23$ & $-0,59 ; 1,97$ & $-11,64 ; 19,87$ \\
\hline 2o:4응 & $-11,36 ; 17,40$ & 0,$86 ; 1,25$ & $-0,39 ; 1,34$ & $-1,84 ; 4,37$ \\
\hline $2 \underline{0}: 5 \underline{0}$ & ne & 0,$93 ; 1,35$ & $-0,30 ; 1,04$ & $-1,64 ; 3,92$ \\
\hline $3 \underline{0}: 40$ & $-11,35 ; 17,53$ & 0,$83 ; 1,22$ & $-0,18 ; 1,56$ & $-2,73 ; 3,34$ \\
\hline $3 \underline{0}: 5 \underline{0}$ & ne & 0,$89 ; 1,31$ & $-0,14 ; 1,20$ & $-2,45 ; 3,00$ \\
\hline $4 \underline{o}: 5 \underline{0}$ & ne & 0,$87 ; 1,29$ & 0,$10 ; 1,45$ & $-1,86 ; 3,66$ \\
\hline
\end{tabular}

ne: não existe nenhum adolescente com baixo peso no $5 \underline{\text { o }}$ quinto de renda familiar per capita. 
Distribuição percentual da população de adolescentes moradores de Niterói, Rio de Janeiro, Brasil, segundo o estado nutricional classificado de acordo com a recomendação da Organização Mundial da Saúde (OMS) 20, por categorias de número de moradores no domicílio *

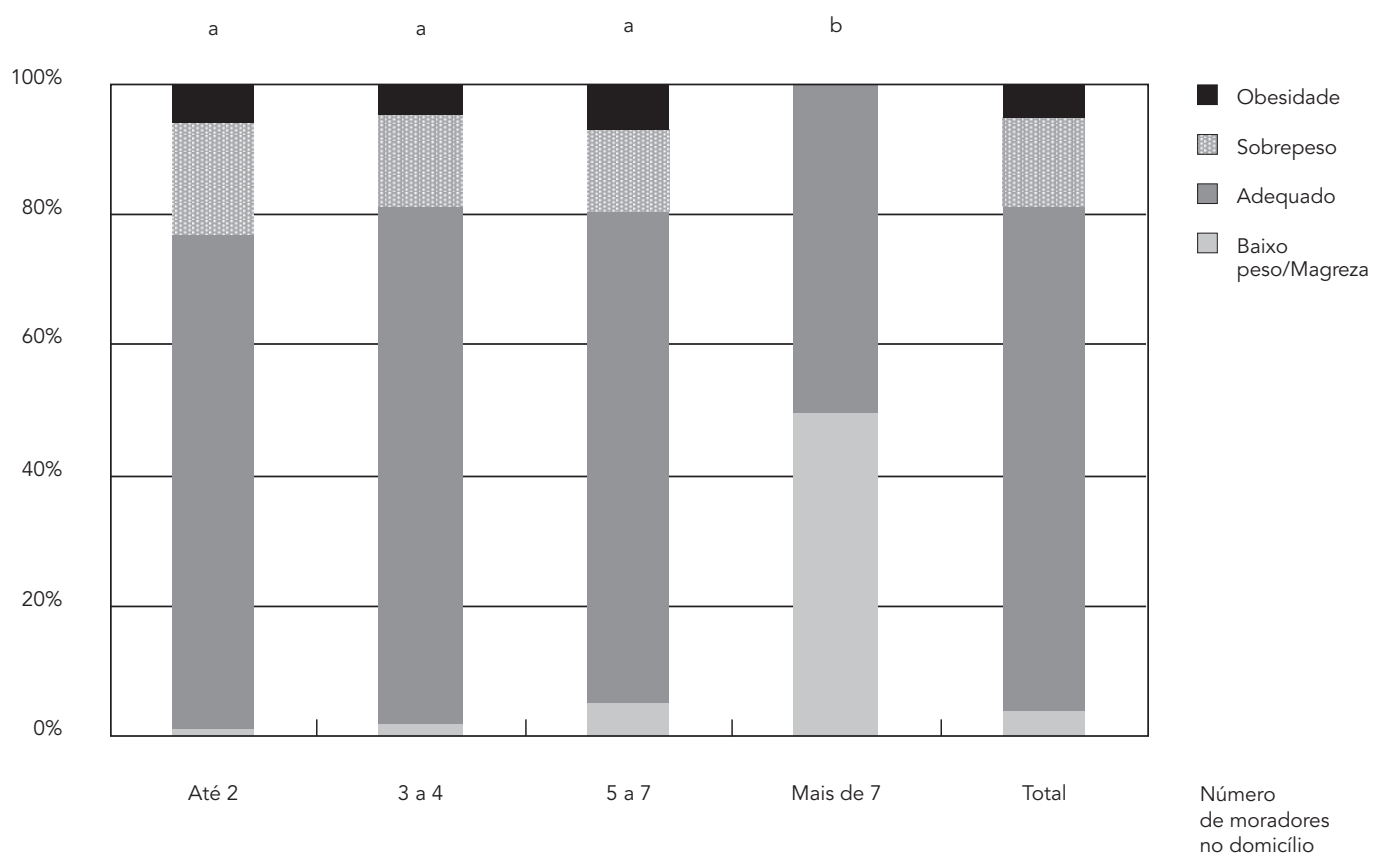

* Na comparação dois a dois entre as classes de números de moradores no domicílio, a mesma letra na parte superior da barra indica que não há diferença significativa $(p>0,05)$ e letras distintas indicam diferenças significativas entre as distribuições proporcionais.

meiras classes (até 2, 3-4 e 5-7), o que pôde ser confirmado pela estatística de Wald para testar a homogeneidade entre os pares de vetores de proporções. Todavia, nos domicílios com mais de sete moradores, não ocorreram adolescentes com sobrepeso ou obesidade, e quase metade $(49,7 \%)$ apresentava baixo peso/magreza, diferindo significativamente das demais distribuições de proporções. Testando a independência desse fator com o estado nutricional, por meio da estatística de Pearson com correções de primeira e segunda ordem, foi identificada associação significativa ( $p<0,001$; sexo masculino: $p<0,05$; sexo feminino: $p<0,001)$. Investigando a associação de cada uma das classes de estado nutricional separadamente com o número de moradores do domicílio, encontrou-se associação positiva significativa entre baixo peso/magreza e número de moradores do domicílio ( $\mathrm{p}<0,01$; sexo masculino: $\mathrm{p}<0,05$; sexo feminino: $\mathrm{p}<0,001$ ).

\section{Discussão}

Os seres humanos desenvolveram mecanismos fisiológicos contra a perda de massa corporal em períodos de escassez de alimentos, mas ainda não existe nenhum mecanismo capaz de prevenir o ganho de massa corporal na presença de alimentos abundantes e/ou inapropriados, situação relativamente recente na história da humanidade. Para explicar a epidemia de obesidade que a população mundial enfrenta atualmente, reconhece-se que deva existir uma interação entre a susceptibilidade biológica e fatores ambientais e sociais que influenciam o quadro 22.

A relação inversa entre sobrepeso/obesidade e situação sócio-econômica é mais evidente entre mulheres adultas 1 . No entanto, vários estudos têm sugerido que essa associação também pode ser encontrada entre adolescentes, de modo que seriam esperadas maiores prevalências de sobrepeso/obesidade entre adolescentes que 
pertencem a famílias de situação sócio-econômica mais desprivilegiadas 23,24,25. Contudo, essa relação ainda é bastante controversa. Turconi et al. ${ }^{26}$, em um estudo no norte da Itália, não encontraram associação significativa. Magalhães \& Mendonça 27 encontraram associação significativa somente entre os meninos, nas regiões Sudeste e Nordeste, apesar de os dados utilizados serem provenientes de uma amostra complexa (pesquisa sobre padrões de vida) e a estatística de teste utilizada $\left(\chi^{2}\right)$ não ter sido corrigida ou ajustada. O mesmo estudo também analisou a associação do número de moradores do domicílio com o estado nutricional, mas não obteve significância. Por outro lado, os resultados do presente estudo demonstram que esses dois fatores encontramse significantemente associados.

Wang et al. ${ }^{14}$, analisando a tendência na prevalência de adolescentes brasileiros com sobrepeso entre as décadas de 1970 e 1990, contrapõem os achados que indicavam uma relação inversa entre a situação sócio-econômica e o sobrepeso/ obesidade, ao demonstrarem que a prevalência de sobrepeso aumentou mais rapidamente nesse período no 3 o terço de renda familiar per capita, tendência que parece ser mais coerente com os achados da presente pesquisa (Figura 1). A investigação de Wang et al. 14 demonstrou, ainda, que o inverso ocorreu nos Estados Unidos, assim como outros estudos descritos anteriormente 24,25 , o que indica que a direção dessa associação pode variar entre países, mediada pelas condições macroeconômicas 7,23. O mesmo foi recentemente evidenciado por Matijasevich et al. 23: com base na comparação entre uma coorte de nascimento britânica e uma brasileira. Os autores identificaram, na coorte brasileira, associação positiva entre a situação sócio-econômica e o sobrepeso/ obesidade em adolescentes; na coorte britânica, a associação foi negativa.

Os resultados da Pesquisa de Orçamentos Familiares (POF), conduzida pelo Instituto Brasileiro de Geografia e Estatística (IBGE) 28 em 20022003, também corroboram essa tendência positiva de maior prevalência de sobrepeso e obesidade em adolescentes nos maiores quintos de renda. Entre as meninas, no 1o quinto (do rendimento monetário mensal familiar per capita), a prevalência de sobrepeso é igual a 9,9\%; no 2 o quinto é $14 \%$; no 3o, $17,2 \%$; no 4o, $16,3 \%$; no 5 o mais rico, $17,6 \%$. Entre os adolescentes do sexo masculino, a prevalência de sobrepeso é igual a $9,3 \%$ no 1o quinto; $14,2 \%$, no $2 \underline{\text { o }} ; 17,8 \%$, no $3 \underline{0} ; 20,6 \%$, no $4 \underline{\text { o }}$; $25,3 \%$, no 5 o 28 . Evidencia-se, dessa forma, que, no Brasil, são encontradas maiores prevalências de sobrepeso em populações de adolescentes com melhores condições financeiras e que, na medida em que os domínios de análise vão se aproximando dos grupos de adolescentes mais pobres, essa prevalência diminui.

Entretanto, é importante ressaltar que, mesmo entre os mais pobres, o sobrepeso representa um problema de saúde pública que atinge aproximadamente 1/10 desse grupo populacional, entre meninos e meninas. Além disso, a comparação dos resultados da POF 2002-2003 28 com estudos realizados pelo IBGE em 1974-1975 (Estudo Nacional da Despesa Familiar) 29 e pelo Instituto Nacional de Alimentação e Nutrição em 1989 (Pesquisa Nacional sobre Saúde e Nutrição) 30 revela importantes aumentos nas prevalências de adolescentes com sobrepeso, inclusive nos quintos de renda mais pobres.

Cabe enfatizar que as prevalências das categorias de estado nutricional estimadas neste estudo segundo os valores de corte atualmente preconizados pela OMS 20 não podem ser diretamente comparadas com as estimativas obtidas por estudos que tenham adotado outros pontos de corte 31 .

\section{Conclusão}

O estado nutricional de adolescentes está associado com a situação sócio-econômica familiar, neste estudo indicada pela renda familiar per capita e pelo número de moradores do domicílio. $\mathrm{O}$ número de moradores do domicílio encontra-se diretamente associado ao baixo peso/magreza em adolescentes. Apesar de não ter sido encontrada associação significativa, há tendência clara de aumento na proporção de adolescentes com sobrepeso/obesidade à medida que os quintos de renda familiar per capita aumentam. É consistente a evidência acerca da esperança de maiores prevalências de adolescentes com sobrepeso/obesidade em grupos de menor status sócioeconômico nos países desenvolvidos. Por outro lado, são necessários mais estudos que permitam confirmar a associação positiva entre a situação sócio-econômica e o sobrepeso/obesidade na adolescência em países em desenvolvimento, como evidenciado por este estudo. 


\section{Resumo}

Investigou-se a relação entre o estado nutricional e a situação sócio-econômica familiar de adolescentes moradores de Niterói, Rio de Janeiro, Brasil. Os dados de 523 adolescentes, pertencentes a uma amostra probabilística de 1.734 domicílios, foram coletados entre janeiro e dezembro de 2003 e representam os 71.922 jovens residentes em Niterói. As análises incluíram a estimação de intervalos de confiança de razões de prevalência e testes de distribuições proporcionais e de independência entre classes de estado nutricional e classes de renda familiar per capita e as faixas de número de moradores do domicílio. Foi encontrada uma associação positiva significativa entre baixo peso/magreza e o número de moradores do domicílio (sexo masculino: $p<0,05$; sexo feminino: $p<0,001)$. O número de moradores do domicílio encontra-se diretamente associado ao baixo peso/magreza em adolescentes. Foi evidenciada uma tendência positiva de aumento na proporção de adolescentes com sobrepeso/obesidade segundo os quintos de renda familiar per capita.

Estado Nutricional; Avaliação Nutricional; Inquéritos Nutricionais; Fatores Sócio-econômicos; Adolescente

\section{Colaboradores}

L. A. Anjos planejou o estudo, supervisionou a coleta de dados de campo e participou das análises estatísticas. M. T. L. Vasconcellos planejou o estudo e a amostra; junto com F. S. Gomes, calculou os pesos amostrais naturais e calibrados e realizou as análises estatísticas. F. S. Gomes participou da coleta de dados e escreveu a primeira versão do manuscrito. L. A. Anjos e M. T. L. Vasconcellos revisaram e aprovaram as versões do manuscrito.

\section{Agradecimentos}

Este estudo contou com o financiamento do Conselho Nacional de Desenvolvimento Científico e Tecnológico (CNPq; processos: 471172/2001-4, 475122/2003-8, 302992/2003-0 e 311801/2006-4) e da Fundação Oswaldo Cruz (Programa Estratégico de Apoio à Pesquisa em Saúde-PAPES III nº. 250.139).

\section{Referências}

1. Monteiro CA, Moura EC, Conde WL, Popkin BM. Socioeconomic status and obesity in adult populations of developing countries: a review. Bull World Health Organ 2004; 82:940-6.

2. Brennan SL, Henry MJ, Nicholson GC, Kotowicz MA, Pasco JA. Socioeconomic status and risk factors for obesity and metabolic disorders in a population based sample of adult females. Prev Med 2009; 49:165-71.

3. Fleischer NL, Diez Roux AV, Alazraqui M, Spinelli H. Social patterning of chronic disease risk factors in a Latin American city. J Urban Health 2008; 85: 923-7.

4. Martín AR, Martínez Nieto JM, Ruiz JPN, Jiménez LE. Overweight and obesity: the role of education, employment and income in Spanish adults. Appetite 2008; 51:266-72.
5. Pikhart H, Bobak M, Malyutina S, Pajak A, Kubínová $\mathrm{R}$, Marmot $\mathrm{M}$. Obesity and education in three countries of the Central and Eastern Europe: the HAPIEE study. Cent Eur J Public Health 2007; 15:140-2.

6. Monteiro CA, Benicio MH, Mondini L, Popkin BM. Shifting obesity trends in Brazil. Eur J Clin Nutr 2000; 54:342-6.

7. Ford ES, Mokdad AH. Epidemiology of obesity in the Western Hemisphere. J Clin Endocrinol Metab 2008; 93(11 Suppl 1):S1-8.

8. Peña M, Bacallao J. La obesidad en la pobreza: un problema emergente en las Américas. In: Peña M, Bacallao J, editores. La obesidad en la pobreza: un nuevo reto para la salud pública. Washington DC: Organización Panamericana de la Salud; 2000. p. 3-11. 
9. Bossan FM, Anjos LA, Vasconcellos MTL, Wahrlich V. Nutritional status of the adult population in $\mathrm{Ni}$ terói, Rio de Janeiro, Brazil: the Nutrition, Physical Activity, and Health Survey. Cad Saúde Pública 2007; 23:1867-76.

10. Cole TJ, Bellizzi MC, Flegal KM, Dietz WH. Establishing a standard definition for child overweight and obesity worldwide: international survey. BMJ 2000; 320:1240-3.

11. World Health Organization. Obesity: preventing and managing the global epidemic. Report of a WHO Consultation on Obesity. Geneva: World Health Organization; 1995. (Technical Report Series, 894).

12. Anjos LA, Castro IRR, Engstrom EM, Azevedo AMF. Crescimento e estado nutricional em amostra probabilística de escolares no Município do Rio de Janeiro, 1999. Cad Saúde Pública 2003; 19 Suppl 1: S171-9.

13. Monteiro CA, Conde WL, Popkin BM. A tendência secular da obesidade segundo estratos sociais: Nordeste e Sudeste do Brasil, 1975-1989-1997. Arq Bras Endocrinol Metab 1999; 43:186-94.

14. Wang Y, Monteiro CA, Popkin BM. Trends of obesity and underweight in older children and adolescents in the United States, Brazil, China, and Russia. Am J Clin Nutr 2002; 75:971-7.

15. World Health Organization. Physical status: the use and interpretation of anthropometry. Report of a WHO Expert Committee. Geneva: World Health Organization; 1995. (Technical Report Series, 854).

16. Lohman TG, Roche AF, Martorell R. Anthropometric standardization reference manual. Champaign: Human Kinetics; 1988.

17. Haldane JBS. On the method of estimating frequencies. Biometrika 1945; 33:222-5.

18. Vasconcellos MTL, Silva PLN, Szwarcwald CL. Sampling design for the World Health Survey in Brazil. Cad Saúde Pública 2005; 21 Suppl 1:S89-99.

19. Silva PLN. Calibration estimation: when and why, how much and how. Rio de Janeiro: Instituto Brasileiro de Geografia e Estatística; 2004. (Textos para Discussão da Diretoria de Pesquisas, 15).

20. Onis M, Onyango AW, Borghi E, Siyam A, Nishida C, Siekmanna J. Development of a WHO growth reference for school-aged children and adolescents. Bull World Health Organ 2007; 85:660-7.
21. Rao JNK, Scott AJ. The analysis of categorical data from complex sample surveys: chi-squared tests for goodness-of-fit and independence in two way tables. J Am Stat Assoc 1981; 76:221-30.

22. Peña M, Bacallao J. Malnutrition and poverty. Annu Rev Nutr 2002; 22:241-53.

23. Matijasevich A, Victora CG, Golding J, Barros FC, Menezes AM, Araujo CL, et al. Socioeconomic position and overweight among adolescents: data from birth cohort studies in Brazil and the UK. BMC Public Health 2009; 9:105.

24. Goodman E, Slap GB, Huang B. The public health impact of socioeconomic status on adolescent depression and obesity. Am J Public Health 2003; 93:1844-50.

25. Vieweg VR, Johnston CH, Lanier JO, Fernandez A, Pandurangi AK. Correlation between high risk obesity groups and low socioeconomic status in school children. South Med J 2007; 100:8-13.

26. Turconi G, Guarcello M, Maccarini L, Bazzano R, Zaccardo A, Roggi C. BMI values and other anthropometric and functional measurements as predictors of obesity in a selected group of adolescents. Eur J Nutr 2006; 45:136-43.

27. Magalhães VC, Mendonça GAS. Prevalência e fatores associados a sobrepeso e obesidade em adolescentes de 15 a 19 anos das regiões Nordeste e Sudeste do Brasil, 1996 a 1997. Cad Saúde Pública 2003; 19 Suppl 1:S129-39.

28. Instituto Brasileiro de Geografia e Estatística. Pesquisa de Orçamentos Familiares 2002-2003: antropometria e análise do estado nutricional de crianças e adolescentes no Brasil. Rio de Janeiro: Instituto Brasileiro de Geografia e Estatística; 2006.

29. Instituto Brasileiro de Geografia e Estatística. Estudo Nacional de Despesas Familiares: 1974-1975. Rio de Janeiro: Instituto Brasileiro de Geografia e Estatística; 1977.

30. Instituto Nacional de Alimentação e Nutrição. Pesquisa Nacional sobre Saúde e Nutrição: resultados preliminares. $2^{\text {a }}$ Ed. Brasília: Instituto Nacional de Alimentação e Nutrição; 1990.

31. Gomes FS, Anjos LA, Vasconcellos MTL. Influence of different body mass index cut-off values in assessing the nutritional status of adolescents in a household survey. Cad Saúde Pública 2009; 25:1850-7.

Recebido em 01/Mai/2009

Versão final reapresentada em 20/Jul/2009

Aprovado em 29/Jul/2009 